\title{
Research Paper \\ Design and Validation of the Equation for Correction of Body Mass Index
}

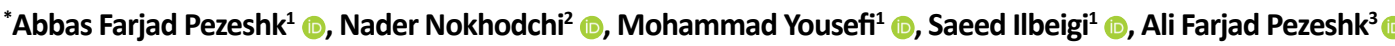

1. Department of Sport Sciences, Faculty of Physical Education and Sport Sciences, University of Birjand, Birjand, Iran.

2. Department of Sport Sciences, Faculty of Human Sciences, University of Bojnord, Bojnord, Iran.

3. Department of Computer Engineering, Faculty of Computer Engineering, Ferdowsi University, Mashhad, Iran.

Citation Farjad Pezeshk A, Nokhodchi N, Yousefi M, Ilbeigi S, Farjad Pezeshk A. Design and Validation of the Equation for Correction of Body Mass Index. Scientific Journal of Rehabilitation Medicine. 2021; 10(3):400-411. https://doi.org/10.32598/sjrm.10.3.2

doi https://doi.org/10.32598/sjrm.10.3.2

Received: 26 Dec 2020 Accepted: 13 Mar 2021 Available Online: 23 Jul 2021

Keywords:

Body Mass Index, Obesity, Somatotypes

\section{A B STR A C T}

Background and Aims The Body Mass Index (BMI) is considered one of the most common indexes for examining the health. The purpose of this study was the design and validation of the equations for the accuracy of BMI.

Methods In the current 419 athletic and non-athletic subjects participated in this study. Anthropometrical measurement was performed using International Standard for Anthropometry and Kinanthropometry (ISAK) protocol, and mesomorphic and endomorphic calculated based on the Heath-Carter methods. The Spearman correlation coefficient was used to determine the relationship between BMI and body fat percent, mesomorphic and endomorphic values. Multiple regressions were used to predict BMI.

Results There is a high correlation between BMI with mesomorphic and endomorphic values $(P<0.01)$, but the correlation of BMI with mesomorphic in athletic men was higher than endomorphic (0.77 vs. 0.63). Multiple regression produces some equations for predicting mesomorphic and endomorphic, and bland Altman believes that all equations predict fatness.

Conclusion According to the results, the Equations proposed in this study could appropriately turn BMI to predict body composition so that this equation could determine higher BMI because of the higher muscular mass.

\section{Extended Abstract}

\section{Introduction}

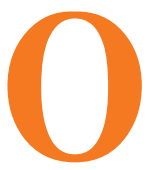

besity is one the most progressing health problems in the current population, in development and underdevelopment countries worldwide. Since there are strong relationships between obesity and health-threatening disease, including diabetes, cancers, and blood pressure, prevention and treating obesity become more important for countries approaching a healthy population. The Body Mass Index (BMI) is considered one of the most common indexes for examination and estimation of the human health situation. However, current research believes that BMI has not enough validity for detecting obesity, especially in the athletic population. It assumed that BMI could not consider inter subjects differences, including sex, activity, and body composition. So it was hypothesized that BMI has less accuracy in predicting obesity in all populations, including athletic and every day. This study aimed to design and validate equations for the correction of the BMI.

\section{${ }^{*}$ Corresponding Author:}

Abbas Farjad Pezeshk, PhD.

Address: Department of Sport Sciences, Faculty of Physical Education and Sport Sciences, University of Birjand, Birjand, Iran.

Tel: +98 (56) 31026915

E-Mail: abbas.farjad@birjand.com 
Table 1. Average and Standard Deviation of mesomorphy, endomorphy, BMI in athletic and non-athletic male and female

\begin{tabular}{ccccc}
\hline \multirow{2}{*}{ Groups } & \multicolumn{3}{c}{ Mean \pm SD } \\
\cline { 2 - 5 } & Mesomorphic Index & Endomorphic Index & Body Mass Index (BMI), $\mathbf{K g} / \mathbf{m}^{2}$ & Body Fat (\%) \\
\hline Athletic men & $4.98 \pm 1.19$ & $4.31 \pm 1.34$ & $22.64 \pm 3.92$ & $20.70 \pm 6.05$ \\
\hline Non-athletic men & $4.85 \pm 2.58$ & $4.79 \pm 2.19$ & $22.57 \pm 3.88$ & $23.64 \pm 10.81$ \\
\hline Athletic women & $4.34 \pm 1.17$ & $7.00 \pm 1.3$ & $22.20 \pm 2.99$ & $35.02 \pm 7.34$ \\
\hline Non-athletic women & $4.22 \pm 1.24$ & $7.00 \pm 1.49$ & $22.15 \pm 2.31$ & $36.43 \pm 8.71$ \\
\hline & & & $\begin{array}{c}\text { Scientific Journal of } \\
\text { Rehabilitation Medicine }\end{array}$ \\
\hline
\end{tabular}

\section{Methods}

In the current study with semi-experimental methods, 419 athletic and non-athletic subjects participated. All subjects have no history of muscle-skeletal disorders, surgery, and walking abnormality that influence their sports activities. Anthropometrical measurement has been performed using International Standard for Anthropometry and Kinanthropometry (ISAK) protocol. For measuring subcutaneous fat, Skinfold fat has been measured using a particular caliper in the following area: triceps on the posterior of the humerus, iliac crest in highest region, mid-thigh in the anterior side of the thigh, subscapularis in the inferior region of scapula, supraspinatus and medial leg in the calf area. For breath and circumference, the breath of humerus and thigh and girth of leg and humerus have been measured. BMI has been calculated using dividing weight by the square of the height. Percent body fat has been calculated using the Peterson equation. Mesomorphic and endomorphic have been calculated based on the Heath-Carter somatotype methods (Table 1). The Spearman correlation coefficient was used to determine the relationship between BMI and body fat percent, mesomorphic and endomorphic values. Multiple regressions were used to predict BMI. For this purpose, mesomorphic and endomorphic are put into the model as dependent variables, and height, weight, and BMI are put into the equation as independent variables. Bland Altman limit of agreement plot was also used to validate the equations extracted by multiple regression. All statistical analyses were done using SPSS software version 16.

\section{Results}

The results of this study have shown that there is a high correlation between BMI with mesomorphic and endomorphic values $(\mathrm{P}<0.01)$. However, the correlation of BMI value with the mesomorphic score in athletic men was higher than this relationship with endomorphic score $(0.77$ vs. 0.63). For multiple regression, mesomorphic and endomorphic variables are put into the model as dependent variables, and height, weight, and BMI are put into the equation as independent variables. Multiple regression has produced some equations for predicting mesomorphic and endomorphic scores, and bland Altman believes that all equations for predicting body fatness and muscularity have an acceptable range of error (Table 2).

\section{Discussion and Conclusion}

This study aimed to design and validate equations for the correction of the Body Mass Index. The results of this study showed that there is a nonlinear relationship between body

Table 2. Regression equations for prediction of obesity and muscularity

\begin{tabular}{ccccccc}
\hline Groups & $\begin{array}{c}\text { Heath-Carter } \\
\text { Correlation }\end{array}$ & SEE & Endomorphic Equations & $\begin{array}{c}\text { Heath-Carter } \\
\text { Correlation }\end{array}$ & SEE & Mesomorphic Equations \\
\hline Athletic men & 0.67 & 0.98 & $\begin{array}{c}20.787+(0.185 \times \mathrm{W})+(- \\
0.135 \times \mathrm{H})+(-0.247 \times \mathrm{BMI})\end{array}$ & 0.85 & 0.61 & $-2.753+(0.533 \times \mathrm{BMI})+(-0.062 \times \mathrm{W})$ \\
\hline $\begin{array}{c}\text { Non-athletic } \\
\text { men }\end{array}$ & 0.76 & 1.40 & $-5.003+(0.434 \times \mathrm{BMI})$ & 0.88 & 0.71 & $-3.284+(\mathrm{BMI} \times 0.504)+(\mathrm{W} \times-0.049)$ \\
$\begin{array}{c}\text { Athletic } \\
\text { women }\end{array}$ & 0.69 & 0.96 & $0.188-(0.040 \times \mathrm{W})+(0.411 \times \mathrm{BMI})$ & 0.82 & 0.67 & $5.261+(0.319 \times \mathrm{BMI})+(0.05 \times \mathrm{H})$ \\
$\begin{array}{c}\text { Non-Athletic } \\
\text { women }\end{array}$ & 0.74 & 0.99 & $-0.146+(0.337 \times \mathrm{BMI})$ & 0.88 & 0.59 & $9.798+(0.287 \times \mathrm{BMI})-(0.75+\mathrm{H})$ \\
\hline
\end{tabular}

$\mathrm{SEE}=$ Standard Error of Estimate.

Scientific Journal of

Rehabilitation Medicine 
fat percent calculated with the Peterson method and BMI in the athletic population. Also, there is a nonlinear relationship between endomorphy value with BMI in the athletic population. It was notable that the relationship between body fat percent calculated with Peterson method and BMI in the athletic population and also between endomorphy value with BMI in the non-athletic group was linear. Based on this finding, BMI is not an appropriate way to assess obesity in the athletic population.

On the other hand, the relationship between mesomorphy score with BMI was linear in the athletic population. This finding indicated that BMI tends to consider the overweight in this athletic group as fatness and obesity; however, it seems that this overweight is because of higher muscular mass in the athletic population. The other purpose of this study was to design equations to predict fatness and muscularity of the athletic population for correction of BMI based on body composition. We used height and weight as dependent variables of the regression model. The same variables were used to calculate BMI. We also used the Bland Altman plot to validate the equation extracted by multiple regression. The Bland-Altman plot has given helpful information regarding the accuracy of the equation in predicting obesity and muscularity. Using the limit of agreement value (Mean diffrences $\pm 2 * \mathrm{SD}$ ), we estimated each equation's upper and lower limits and plotted them. The Limit of the agreement for mesomorphy in four groups was 1.11 (average in four groups) that is acceptable concerning input variables(only weight and height). The limit of agreement for endomorphy in the four groups was 1.8. Besides of high score, concerning the aim of the study, it seems that it was an acceptable value to predict the tendency of body composition. According to the results, the Equations proposed in this study could appropriately turn BMI to predict body composition so that this equation could determine higher BMI because of the higher muscular mass. So the results of this study could be used by athletes, coaches, and clinicians to measure the exact amount of body fatness and obesity using the BMI index.

\section{Ethical Considerations}

\section{Compliance with ethical guidelines}

This study was approved by the Ethics Committee of Kharazmi University (Code: 141/1000/KAP).

\section{Funding}

This research did not receive any grant from funding agencies in the public, commercial, or non-profit sectors.

\section{Authors' contributions}

Authors contributed equally in preparing this article.

\section{Conflict of interest}

The authors declared no conflict of interest.

Acknowledgments 


\section{طراحى و اعتباريابى معادلاتى براي افزايش صحت شاخص توده بدنى}

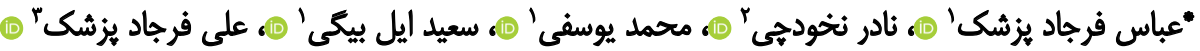

1. كروه علوم ورزشى، دانشكده تربيتبلنى و علوم ورزشى، دانشعاه بيرجئل، بيرجئد، ايران.

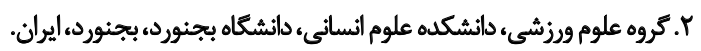

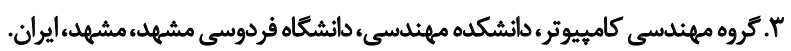

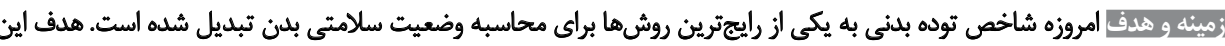

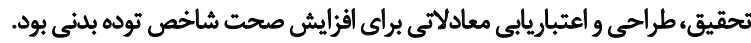

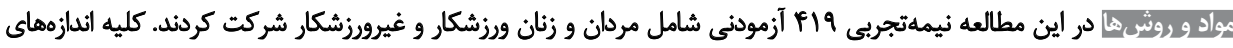

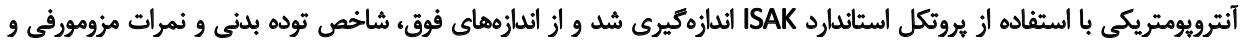

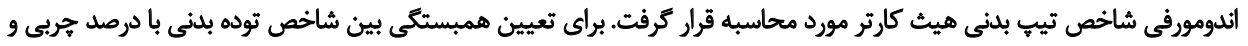

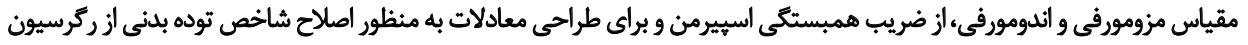

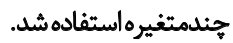

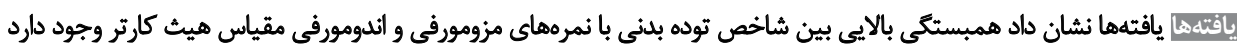

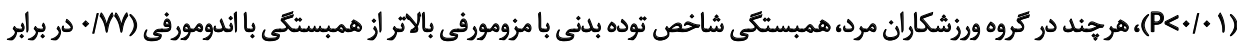

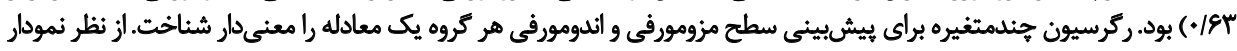

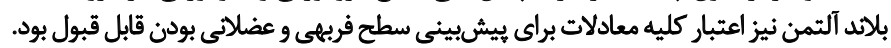

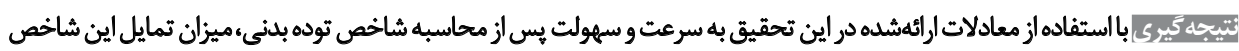

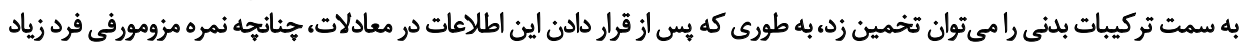

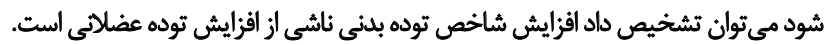

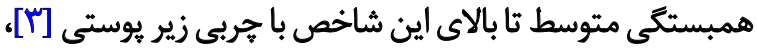

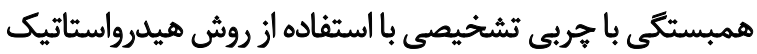

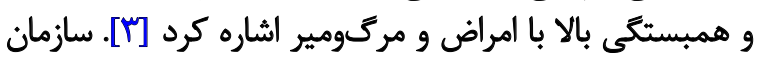

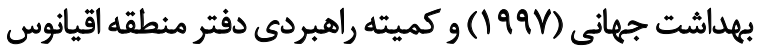

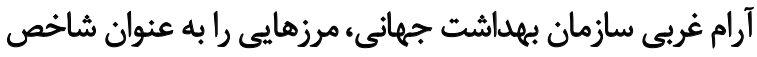

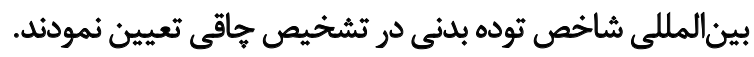

در حال حاضر برخى تحقيقات نشان مي دهنيد شاخص تودئ

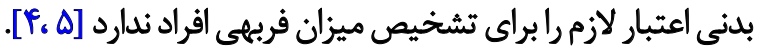

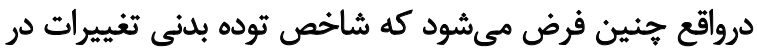

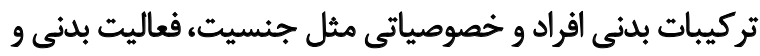

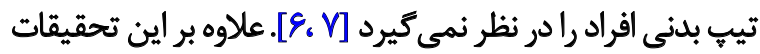

هاقي يكى از رو به رشدترين تهديدات براى سلامتى در دنيا،

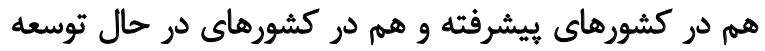

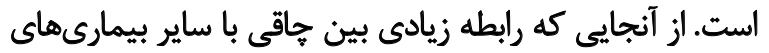

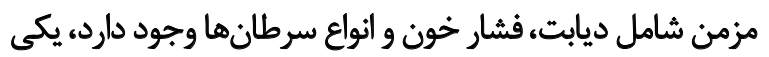

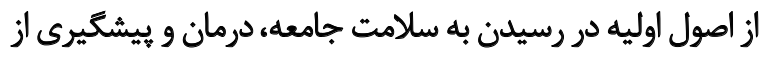

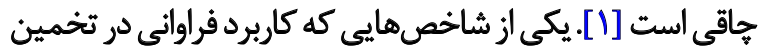

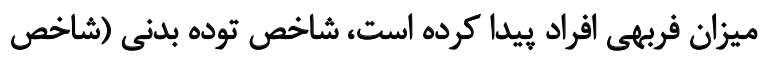

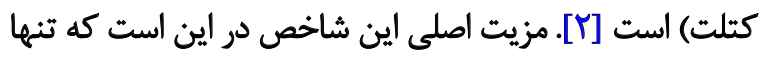

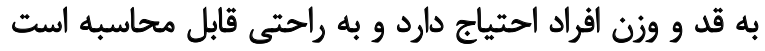

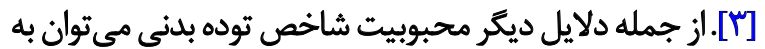


آزمون ورودى دانشكاه و با رضايت شركت كنندكان و ذكر اين

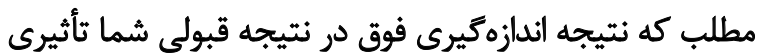

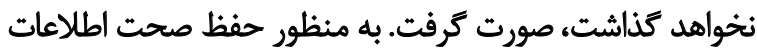

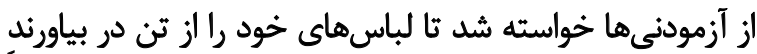

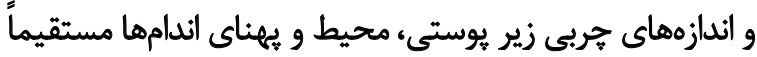

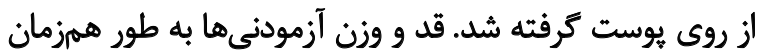

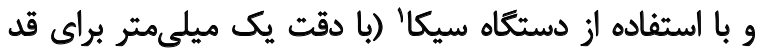

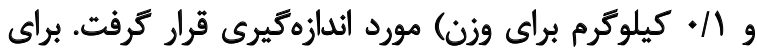

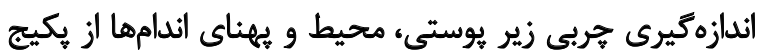

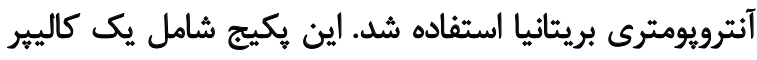

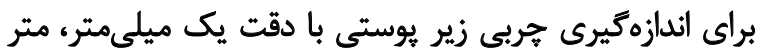

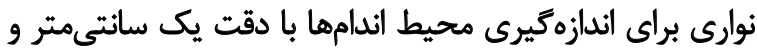

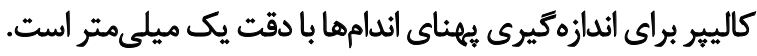

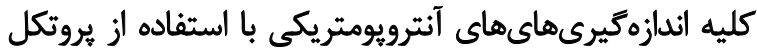

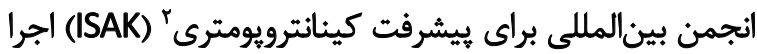

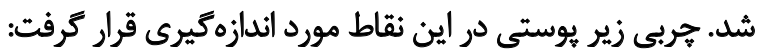

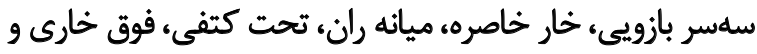

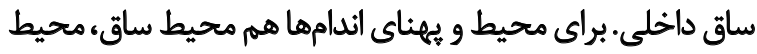

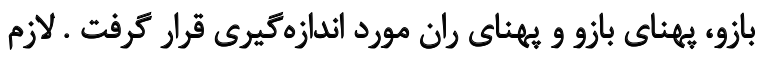

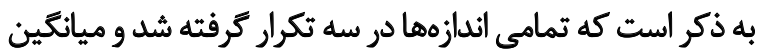
تكرارها به عنوان ركورد آزمودنى ثبت شاند.

براى تعيين شاخص توده بدنى آزمودنى ها، معادله كتلت

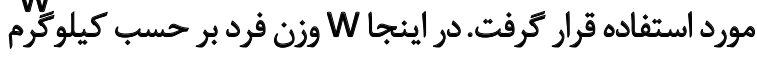

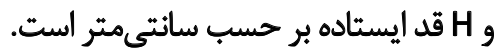

براى تعيين نمرههاى مزومورفى و اندومورفى افراد از مقياس

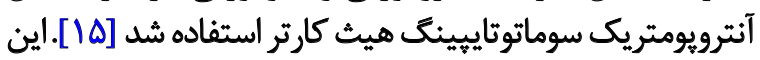

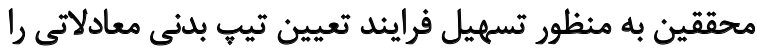

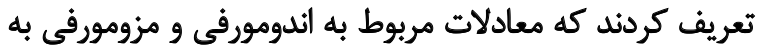

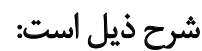

\section{Endomorphy $=-0.7182+01451 X-0.00068 X^{2}+$ $0.000014 X^{3}$}

در اينجا مجموع حربى زير يوستى نواحى فوق خارى، سهسر بازويى و تحت كتفى است.

\section{Mesomorphy $=0.858 \mathrm{HB}+0.601 \mathrm{FB}+0.188 \mathrm{AG}+$ $0.161 C G-0.131 S H+4.5$}

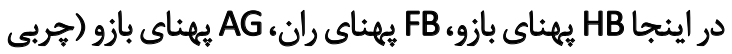

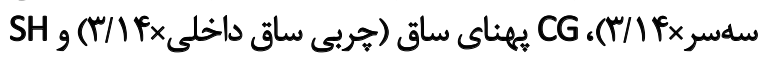
قد ايستاده است.

براي محاسبه درصد جربى بدن از معادله بيترسون و همكاران

1. Seca. Model 755. Germany. CE 0123.

2. International Society for the Advancement of Kinanthropometry (ISAK)
كندشته نشان دادهاند كه شاخص توده بدنى اغلب در تشخيص

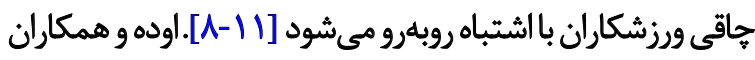

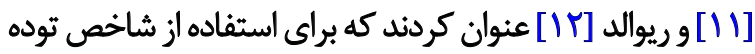

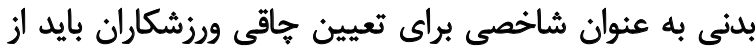

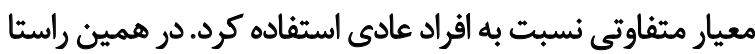

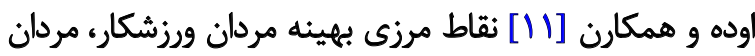

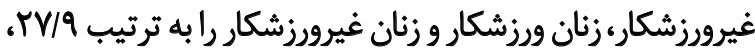

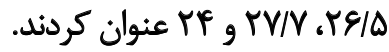

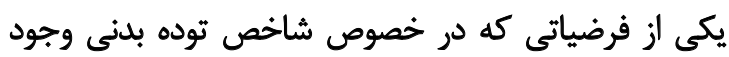

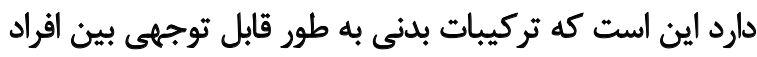

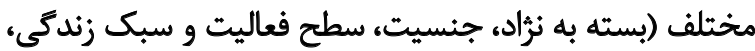

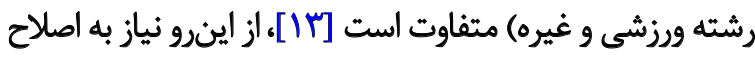

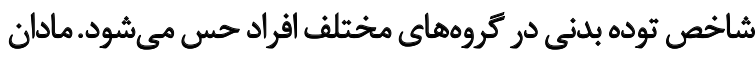

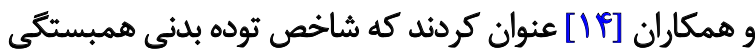

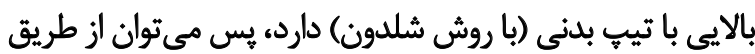

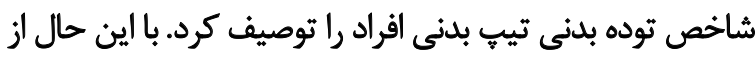

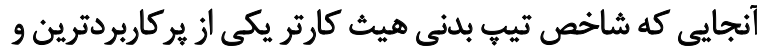

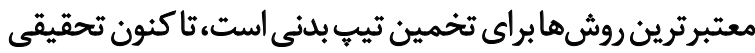

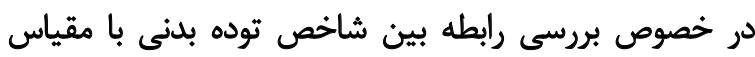

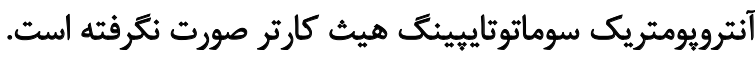

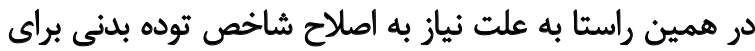

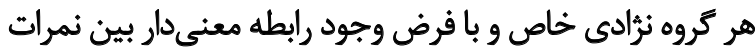

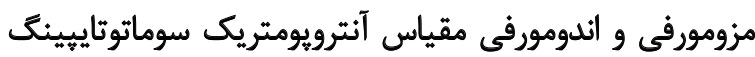

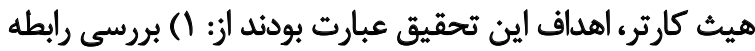

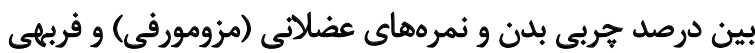

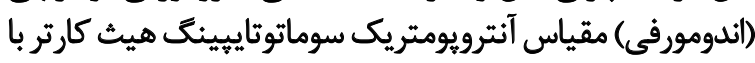

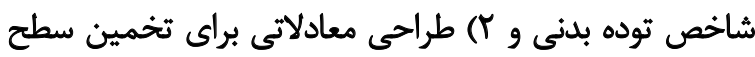

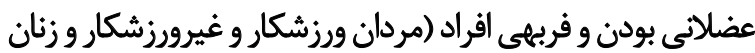

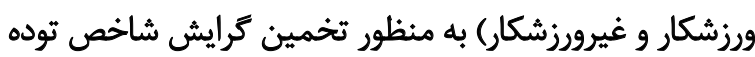

$$
\text { مواد و ورونى سمت تركيبات بدنى. }
$$

جامعه آمارى اين تحقيق را افراد متقاضى ورود به رشتئه

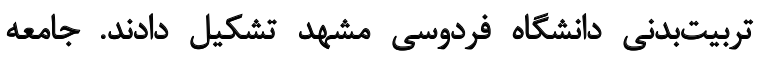

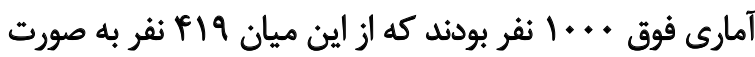

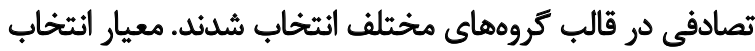

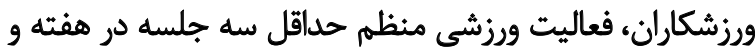

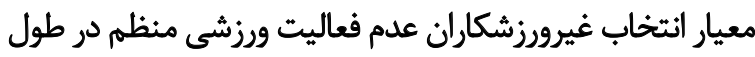

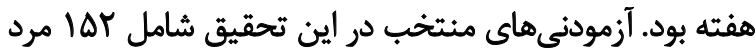

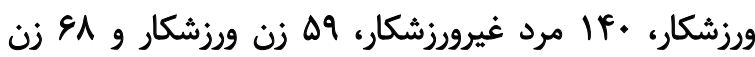

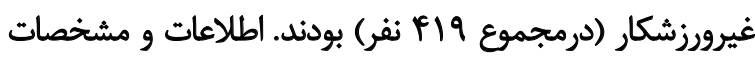
فردى آزمودنى ها در جدول شموع شماره الرائه شده است.

كليه مراحل جمعآورى اطلاعات در قالب تست بزشكى 
جدول ا. ميانكين و انحراف استاندارد سن، قد، وزن و شاخص توده بدنى آزمودنىها

\begin{tabular}{|c|c|c|c|c|}
\hline \multicolumn{4}{|c|}{ ميانكين+انحرافمعيار } & \multirow{2}{*}{ تمروه } \\
\hline شاخص توده بدنى (كيلوكرم بر متر مربع) & 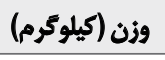 & قد (ساتثىمتر) & سن (سال) & \\
\hline rr/grtm/ar & ENTq土1.\%. & $\mid W N / \circ A \pm N \&$ & $r \cdot M \cdot \pm F / M$ & مران ورزشكار \\
\hline$r r / \Delta \Lambda \pm r / M$ & \&NYA $\pm I \Psi / F \Delta$ & $\mid W^{2} / q \mp \pm \Delta / M$ & $r \cdot / V \Delta \pm r / N \Delta$ & مردان غيرورزشكار \\
\hline$r T / \Psi \cdot \pm T / 99$ & $\Delta V / \backslash \pm \pm \sqrt{ } /{ }_{1} \mid$ & $|E \cdot M| \pm \Delta / M$ & $|N / E q \pm Y / A|$ & زنان ورزشكار \\
\hline$M / / \Delta \pm \Psi / m \mid$ & $\Delta \Delta / T Y \pm N I I$ & $1 Q N E E \pm \triangle / T \Delta$ & $19 / \Delta A \pm 1 / Q C$ & زنان غيرورزشكار \\
\hline
\end{tabular}

bُ إن

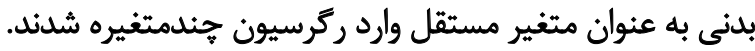

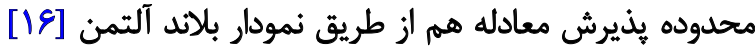

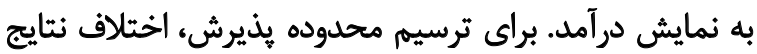

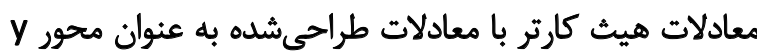

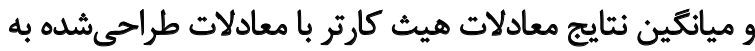

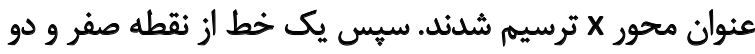

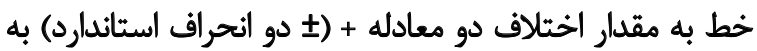

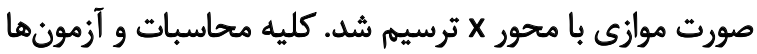

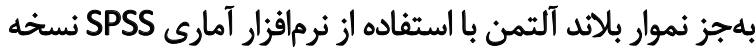

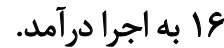

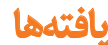

ميانكين و انحراف استاندارد نمرات مزومورفى، اندومورفى، دورئ

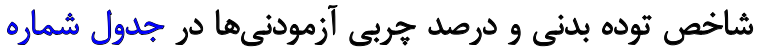

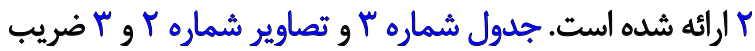

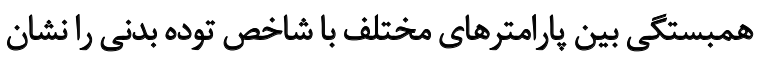

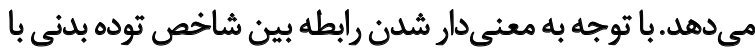

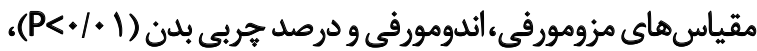

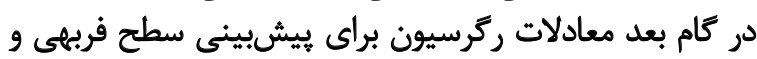

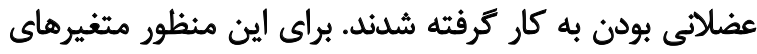

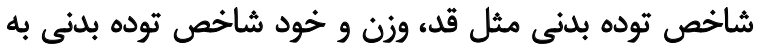

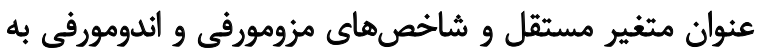
عنوان متغير وابسته وارد مدل ركرسيون شدند. مزوماند

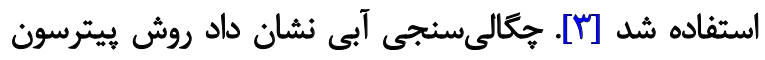

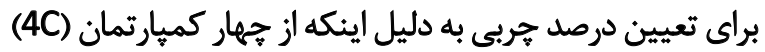

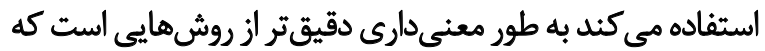

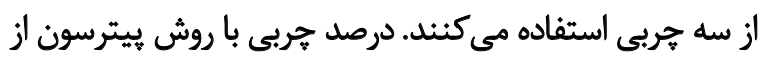

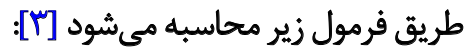

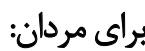

$\%$ Fat $=20.94878+($ agex0.1166) $-(H t \times 0.11666)+$ $(\Sigma 4 \times 0.42696)-\left(\Sigma 4^{2} \times 0.00159\right)$

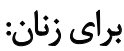

$$
\begin{gathered}
\% F a t=22.18945+(\text { agex0.06368) }+(B M I x \\
0.60404)-(H t \times 0.14520)
\end{gathered}
$$

$$
+(\Sigma 4 \times 0.30919)-\left(\Sigma 4^{2} \times 0.00099562\right)
$$

در اينجا age= سن، Ht= قد، شاخص توده بدنى= شاخص كتص

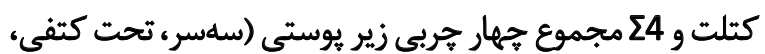
فوق خارى و ران ميانى) است.

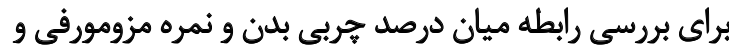

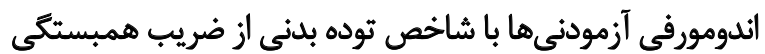

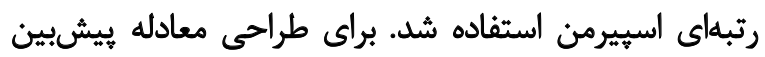

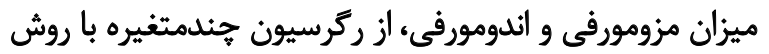

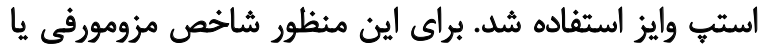

\begin{tabular}{|c|c|c|c|c|}
\hline \multicolumn{4}{|c|}{ ميانكين +انحرافمعيار } & \multirow{2}{*}{ كمووه } \\
\hline درصد هربى بدن & (كيلوكرمبر توده بدئم) & شاخص اندومورفى & شاخص مزومورفى & \\
\hline$r+N \cdot \pm F /+\Delta$ & $r r / g F \pm T / q T$ & $\varphi / \Psi \pm 1 / \Psi \varphi$ & $r / u \pm v / 1 q$ & مردان ورزشكار \\
\hline$\pi / 9 \mp \pm 1 \cdot \mid \Lambda 1$ & $T r / \Delta V \pm T / M$ & $r / M Q T / / q$ & $F / \Delta \Delta \pm Y / \Delta A$ & مردان غيرورزشكار \\
\hline$r \Delta / \cdot r \pm V / / 4 r$ & $T r / T \cdot \pm r / q q$ & $\mathrm{~V} / \cdot . \pm \mathrm{V} / \mathrm{r}$ & $r / T Y \pm V / / Y$ & زنان ورزشكار \\
\hline$r g / P T \pm \mathcal{N} n$ & $r / N \Delta \pm T / M I$ & $V / . . \pm V / p q$ & $r / / T \pm V / Y r$ & زنان غيرورزشكار \\
\hline
\end{tabular}

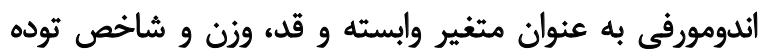

جدول ז. ميانكين و انحراف استاندارد شاخصهاى مزومورفي، اندومورفي، شاخص توده بدنى (شاخص توده بدنى) مردان و زنان ورزشكار و مردان و زنان غيرورزشكار 
جدول ب. ضريب همبستكى اسييرمن بين شاخص توده بلدى با درصد جربى بلن، مزومورفى و اندومورفى مردان و زنان ورزشكار و غيرورزشكار

\begin{tabular}{|c|c|c|c|}
\hline درصد هريس بدن & اندومورفى & مزومورفى & كروه \\
\hline .181 & .198 & $\cdot / 1$. & هردان ورزشكار \\
\hline$\cdot / 1$. &.$/ N F$ & $\cdot / N$ & مردان غيرورزشكار \\
\hline .180 & $.18 v$ & 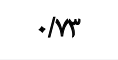 & زنان ورزشكار \\
\hline.$/ v 8$ & ./va & 年 & زنان غيرورزشكار \\
\hline
\end{tabular}

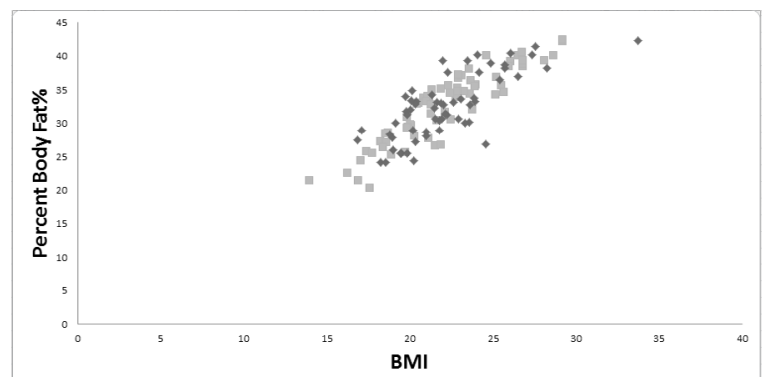

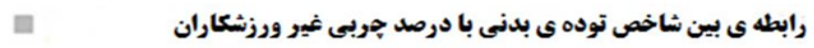

$\bullet$

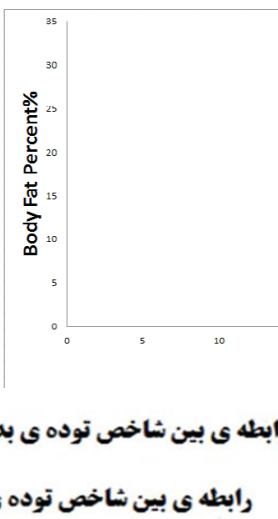

تصوير ا. رابطه بين شاخص توده بدني با درصد جربي بدرن

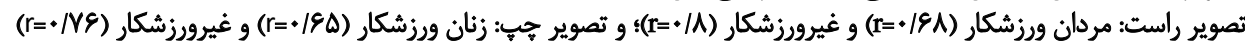

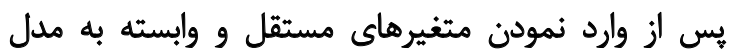

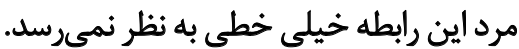

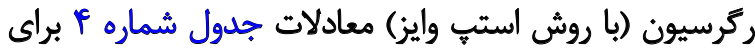

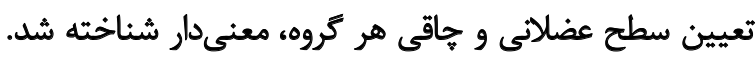

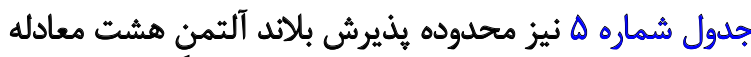
رانشان ميدهد.

اهداف اين تحقيق، بررسى رابطه بين درصد جربى بدن و

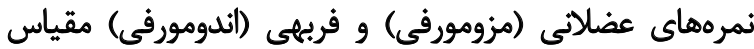

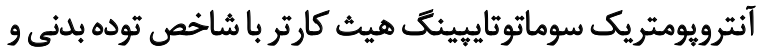

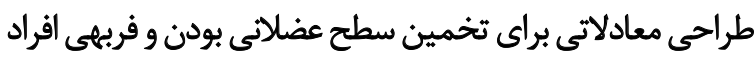
(مردان ورزشكار و غيرورزشكار و زنان ورزشكار و غيرورزئشيكار)

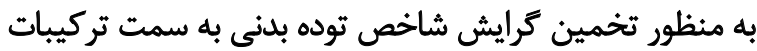

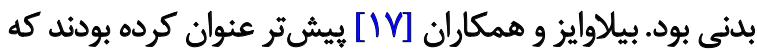

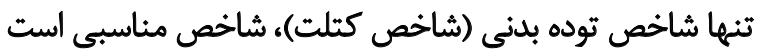

جدول f. ليست معادلات ركرسيون براى تخمين سطح فربهي و توسعه عضلاني بودن ورزشكاران و غيرورزشكاران زن و مرد

\begin{tabular}{|c|c|c|c|c|c|c|}
\hline همبستكي كارتي با & SEE & معادله اثدومورفى & هيبت كارتئ با & SEE & معادله مزومورفى & 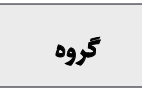 \\
\hline .198 &.$/ \mathrm{H}$ & $\begin{array}{c}20.787+(0.185 \times \mathrm{W})+(- \\
0.135 \times \mathrm{H})+(-0.247 \times \mathrm{BMI})\end{array}$ & . /AS & .181 & $-2.753+(0.533 \times \mathrm{BMI})+(-0.062 \times \mathrm{W})$ & مرد موزشكار \\
\hline.$/ \mathrm{FF}$ & $1 / f^{2}$ & $-5.003+(0.434 \times \mathrm{BMI})$ &.$/ M$ & $\cdot M$ & $-3.284+(\mathrm{BMI} \times 0.504)+(\mathrm{W} \times-0.049)$ & مرد غيرورزشكار \\
\hline leq &.$/ 98$ & $0.188-(0.040 \times \mathrm{W})+(0.411 \times \mathrm{BMI})$ & - /Ar &.$/ E V$ & $5.261+(0.319 \times \mathrm{BMI})+(0.05 \times \mathrm{H})$ & 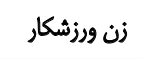 \\
\hline$\cdot M$ &.$/ 99$ & $-0.146+(0.337 \times \mathrm{BMI})$ & $\cdot / M$ &.$/ 189$ & $9.798+(0.287 \times \mathrm{BMI})-(0.75+\mathrm{H})$ & ز ت غيرورزشكار \\
\hline
\end{tabular}

در نمودارهاى موجود در تصوير شماره ا بله خوبى مشخص

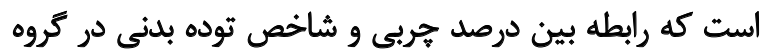

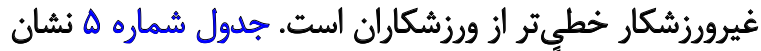

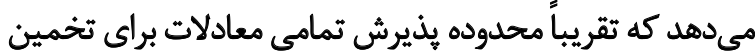

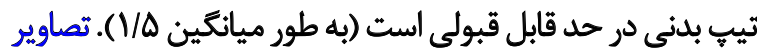

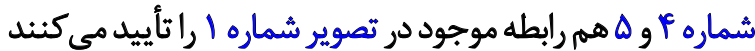
و نشان مي دهند كه بين شاخص توده بدني با نمرات اندومورفي 
جدول هـ محدوده يذيرش بلاند آلتمن معادلات تخمين مزومورفى و اندومورفى براى مردان و زنان ورزشكار و غيرورزشكار

\begin{tabular}{|c|c|c|}
\hline هحدوده هذيرش" اندومورفى & محدوده يذيرش" مزومورفى & كروه \\
\hline 1/9. & V/TE & \multirow{2}{*}{ مرد ورزشكار } \\
\hline$-1 / 99$ & $-1 / 19$ & \\
\hline V/AV & $V / r$ & \multirow{2}{*}{ مرد غيرورزشكار } \\
\hline $1 / M$ &.$- / 99$ & \\
\hline V/qu & $1 / 18$ & \multirow{2}{*}{ زن ورزشكار } \\
\hline$-1 / 19$ & $-1 / \pi$ & \\
\hline 1/9. & ./u & \multirow{2}{*}{ ن غن غيرورزنكار } \\
\hline$-1 / A \Delta$ & $-1 / / r$ & \\
\hline
\end{tabular}

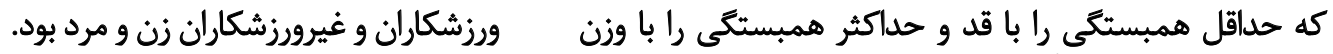
در تروه غيرورزشكار، نتايج اين تحقيق، نتايج تحقيق كذشته

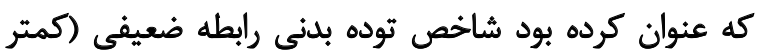

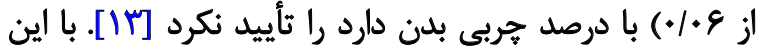

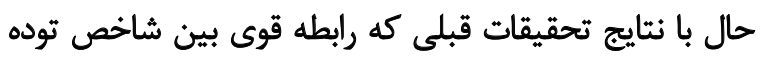

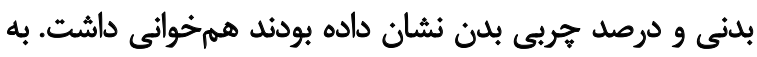

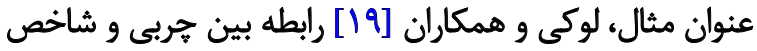

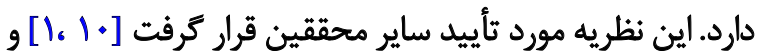

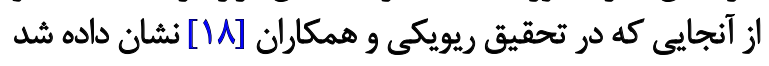

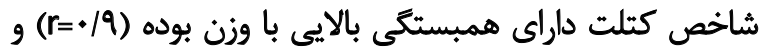

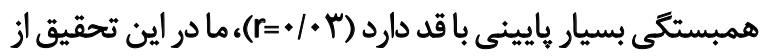

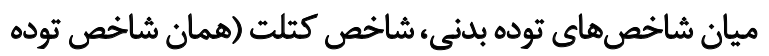

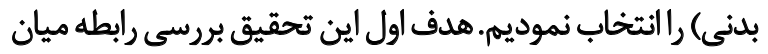

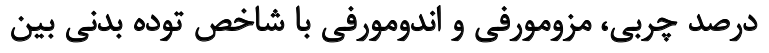
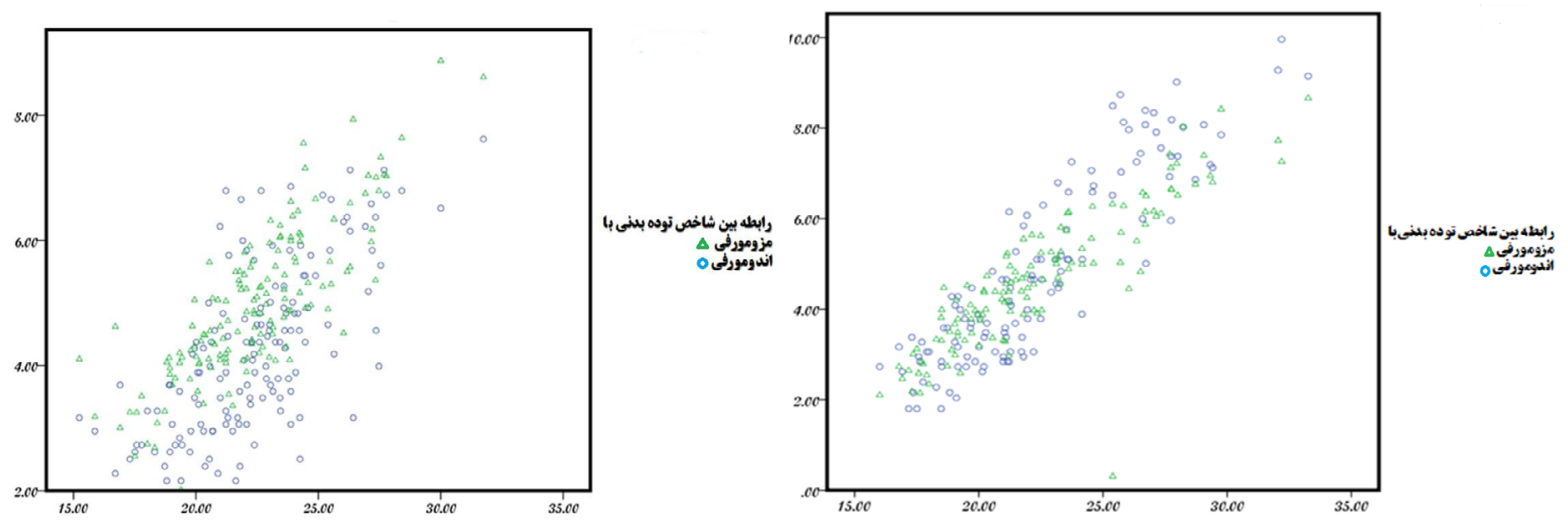

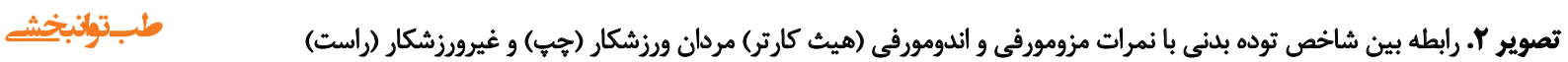
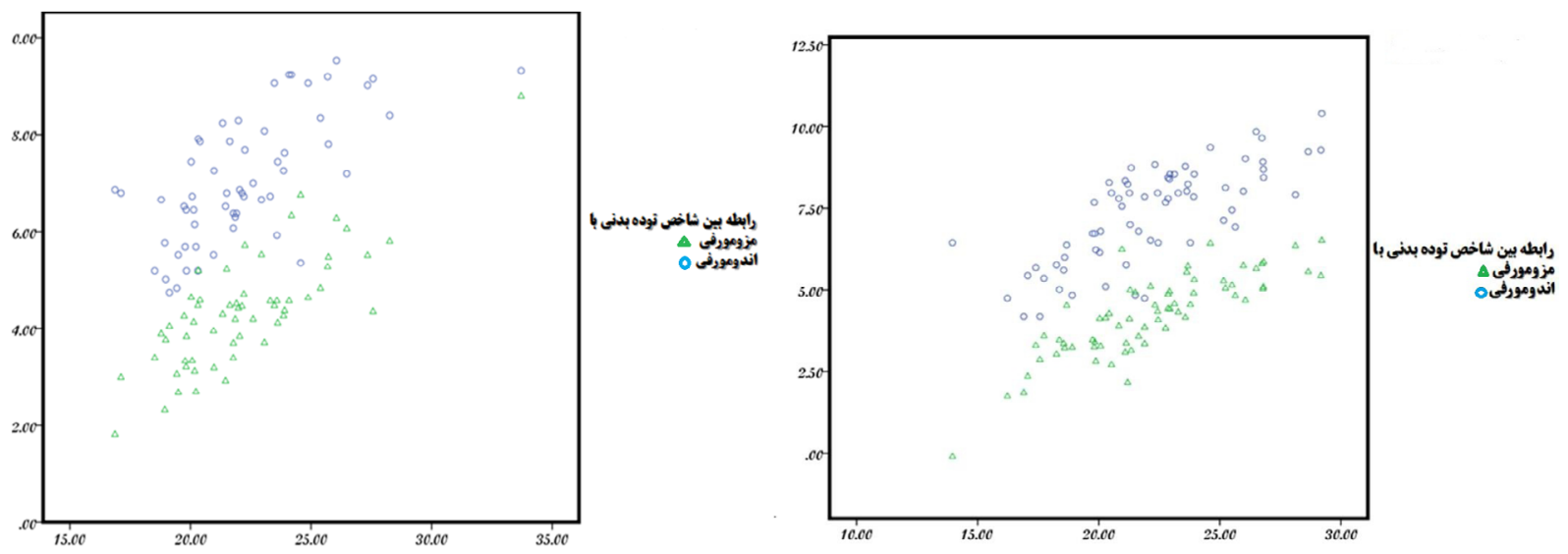

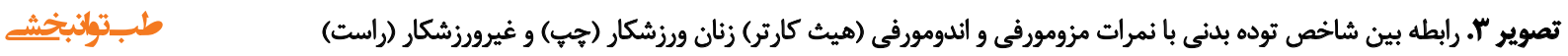




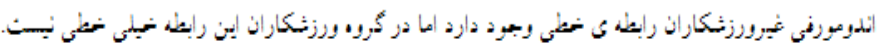

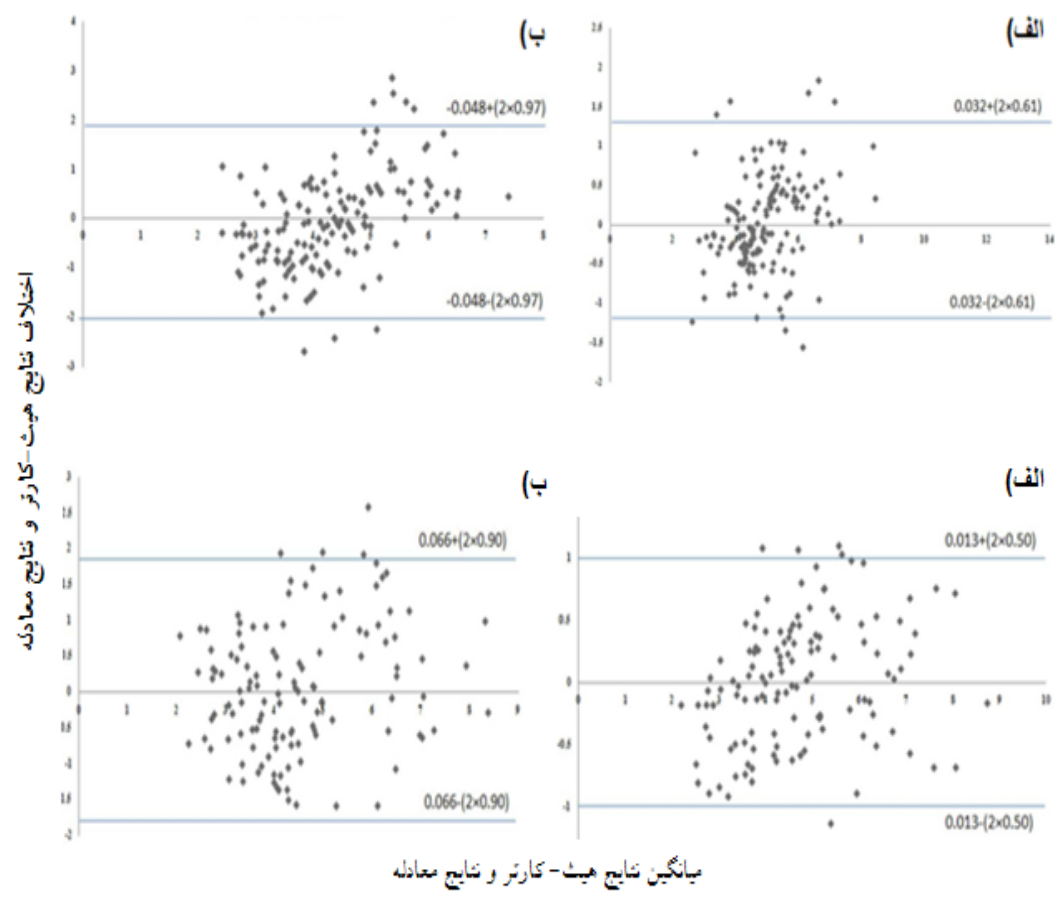

تصوير f. نمودار بلاند آلتمن براى محدوده يذيرش معادله مزومورفى (الف) و اندومورفى (ب) مردان غيرورزشكار (بالاع) و ورزشكار (يايين)

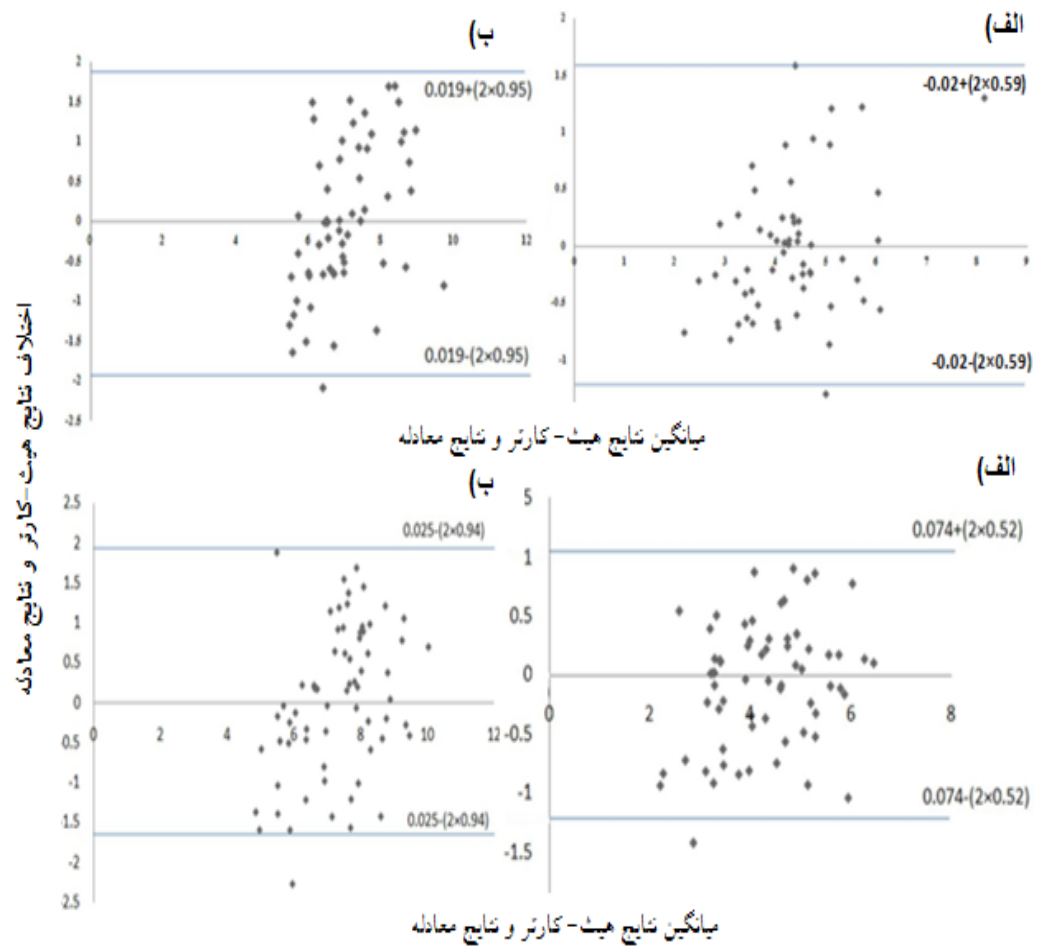

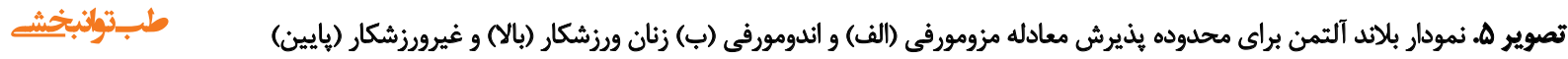
توده بدنى در جنس ها و نثرادهاى مشابه را قوى توصيف كرده 


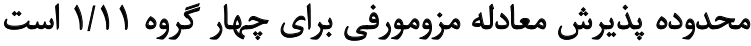

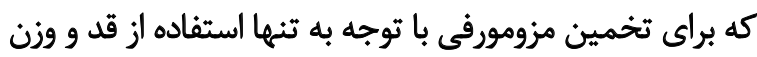

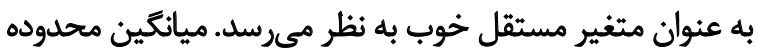

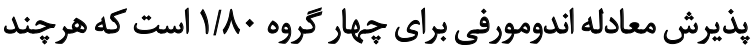

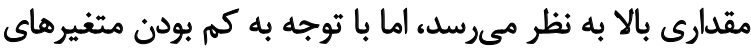

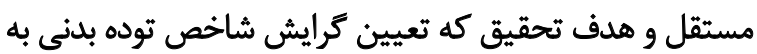
تركيبات بدنى است قابل قبول خواهد بون بودي

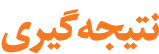

نتايج اين مطالعه نشان داد شاخص توده بدنى در تشخيص

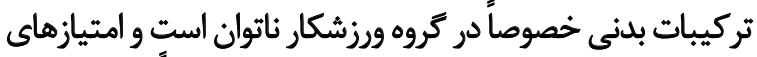

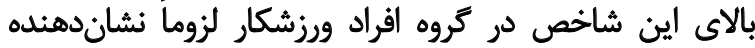

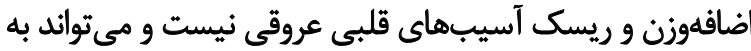

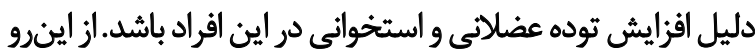

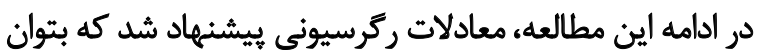

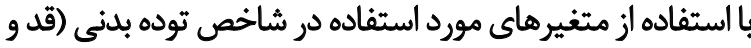

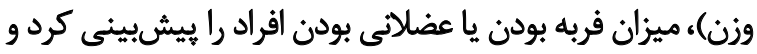

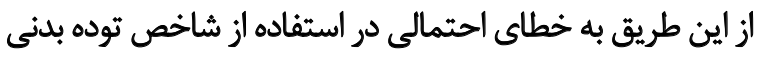

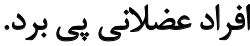

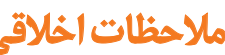

\section{يبيووى أز اصول اخمالاق يُؤشش}

در اجراى يُروهش، ملاحظات اخلاقى مطابق با دستورالعمل

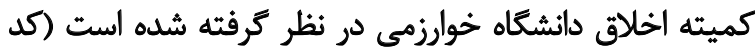

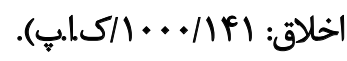

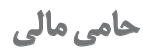

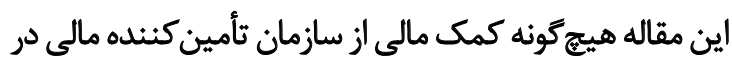

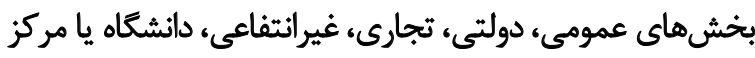

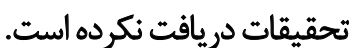

$$
\text { مشاركت نويسند مكان }
$$

تمام نويسندكان در آمادهازى اين مقاله مشاركت يكسان

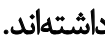

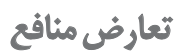

بنابر اظهار نويسندكان اين مقاله تعارض منافع ندارد.
بودند (r=/9). در مطالعه ديكر عنوان شد كه شاخص توده

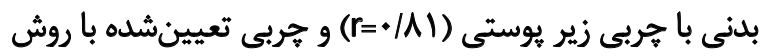

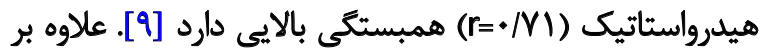

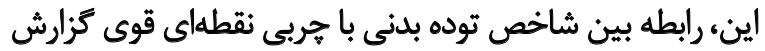

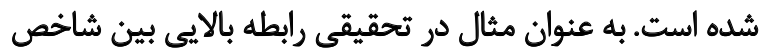

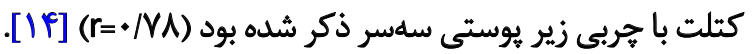

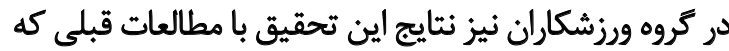

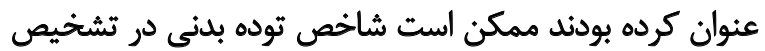

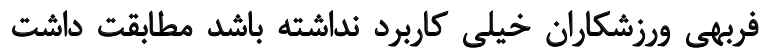

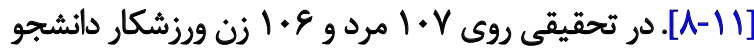

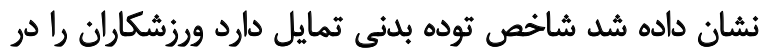

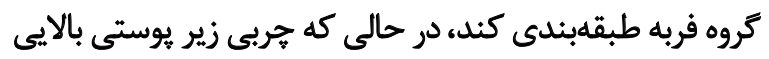

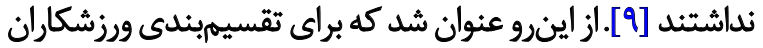

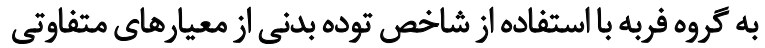

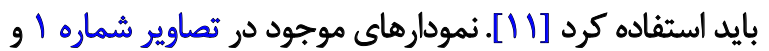

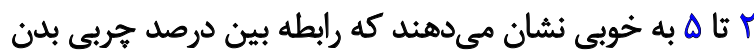

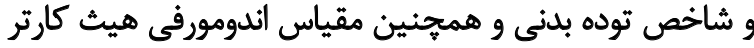

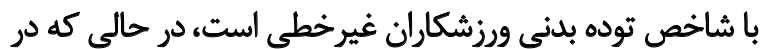

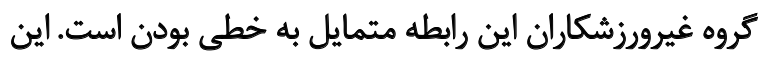

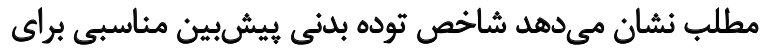

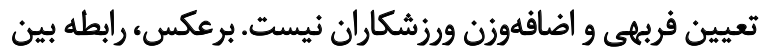

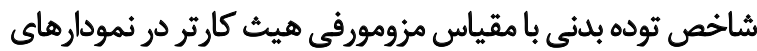

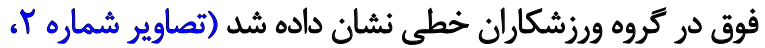

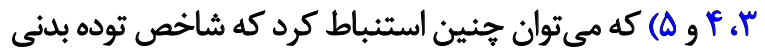

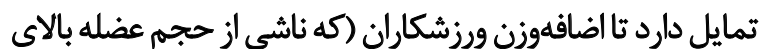
آنهاست) رابه افزايش جربى نسبت بدرندان

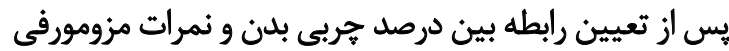

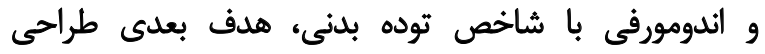

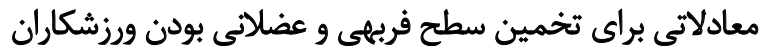

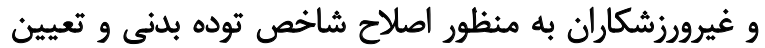

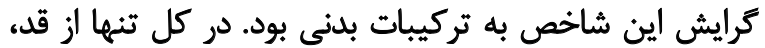

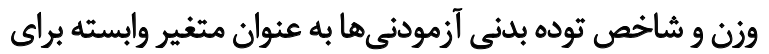

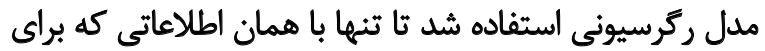

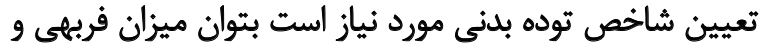

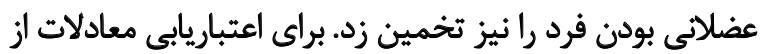

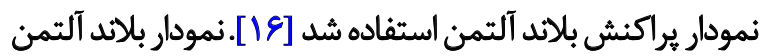

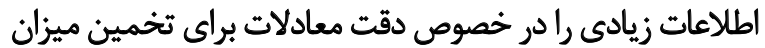

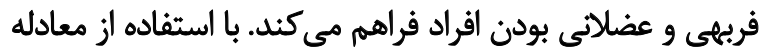

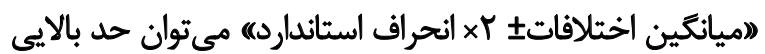

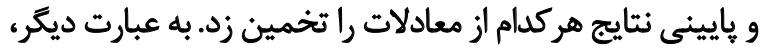

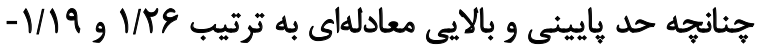

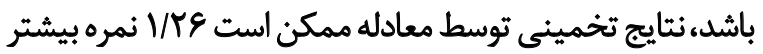
از نمره واقعى يا 9 //1 - نمره باييينتر از نمره واقعى شود. ميائكين 


\section{References}

[1] Kagawa M, Kerr D, Uchida H, Binns CW. Differences in the relationship between $\mathrm{BMI}$ and percentage body fat between Japanese and Australian-Caucasian young men. British Journal of Nutrition. 2006; 95(5):1002-7. [DOI:10.1079/BJN20061745] [PMID]

[2] Jackson AS, Stanforth P, Gagnon J, Rankinen T, Leon AS, Rao D, et al. The effect of sex, age and race on estimating percentage body fat from body mass index: The heritage family study. International Journal of Obesity. 2002; 26(6):789-96. [DOI:10.1038/ sj.ijo.0802006] [PMID]

[3] Eston R, Reilly T. Kinanthropometry and exercise physiology laboratory manual: Tests, procedures and data: Volume two: Physiology. $3^{\text {th }}$ ed. England: Routledge; 2013. [DOI:10.4324/9780203868737]

[4] Kyle UG, Schutz Y, Dupertuis YM, Pichard C. Body composition interpretation: contributions of the fat-free mass index and the body fat mass index. Nutrition. 2003; 19(7-8):597-604 . [DOI:10.1016/S0899-9007(03)00061-3]

[5] Frankenfield DC, Rowe WA, Cooney RN, Smith JS, Becker D. Limits of body mass index to detect obesity and predict body composition. Nutrition. 2001; 17(1):26-30. [DOI:10.1016/S08999007(00)00471-8]

[6] Wagner DR, Heyward VH. Measures of body composition in blacks and whites: A comparative review. The American Journal of Clinical Nutrition. 2000; 71(6):1392-402. [DOI:10.1093/ ajcn/71.6.1392] [PMID]

[7] Prentice AM, Jebb SA. Beyond body mass index. Obesity Reviews. 2001; 2(3):141-7. [DOI:10.1046/j.1467-789x.2001.00031.x] [PMID]

[8] Witt KA, Bush EA. College athletes with an elevated body mass index often have a high upper arm muscle area, but not elevated triceps and subscapular skinfolds. Journal of the American Dietetic Association. 2005; 105(4):599-602. [DOI:10.1016/j. jada.2005.01.008] [PMID]

[9] Kraemer WJ, Torine JC, Silvestre R, French DN, Ratamess NA Spiering BA, et al. Body size and composition of National Football League players. Journal of Strength and Conditioning Research. 2005; 19(3):485-9. [DOI:10.1519/18175.1] [PMID]

[10] Nevill AM, Stewart AD, Olds T, Holder R. Relationship between adiposity and body size reveals limitations of BMI. American Journal of Physical Anthropology. 2006; 129(1):151-6. [DOI:10.1002/ ajpa.20262] [PMID]

[11] Ode JJ, Pivarnik JM, Reeves MJ, Knous JL. Body mass index as a predictor of percent fat in college athletes and nonathletes. Medicine \& Science in Sports \& Exercise. 2007; 39(3):403-9. [DOI:10.1249/01.mss.0000247008.19127.3e] [PMID]

[12] Riewald S. Does the body mass index accurately reflect percent body fat in athletes? Strength \& Conditioning Journal. 2008; 30(1):80-1. [DOI:10.1519/SSC.0b013e318163bc23]

[13] Gallagher D, Heymsfield SB, Heo M, Jebb SA, Murgatroyd PR, Sakamoto $Y$. Healthy percentage body fat ranges: An approach for developing guidelines based on body mass index. The American Journal of Clinical Nutrition. 2000; 72(3):694-701. [DOI:10.1093/ ajcn/72.3.694] [PMID]
[14] Maddan S, Walker JT, Miller JM. Does size really matter? A reexamination of Sheldon's somatotypes and criminal behavior. The Social Science Journal. 2008; 45(2):330-44. [DOI:10.1016/j.soscij.2008.03.009]

[15] Heath BH, Carter JE. A modified somatotype method. American Journal of Physical Anthropology. 1967; 27(1):57-74. [DOI:10.1002/ajpa.1330270108] [PMID]

[16] Bland JM, Altman D. Statistical methods for assessing agreement between two methods of clinical measurement. The Lancet. 1986; 327(8476):307-10. [DOI:10.1016/S01406736(86)90837-8]

[17] Billewicz W, Kemsley W, Thomson A. Indices of adiposity. British Journal of Preventive \& Social Medicine. 1962; 16(4):183-8. [DOI:10.1136/jech.16.4.183] [PMID] [PMCID]

[18] Revicki DA, Israel RG. Relationship between body mass indices and measures of body adiposity. American Journal of Public Health. 1986; 76(8):992-4. [DOI:10.2105/AJPH.76.8.992] [PMID] [PMCID]

[19] Luke A, Durazo-Arvizu R, Rotimi C, Prewitt TE, Forrester T, Wilks $R$, et al. Relation between body mass index and body fat in black population samples from Nigeria, Jamaica, and the United States. American Journal of Epidemiology. 1997; 145(7):620-8. [DOI:10.1093/oxfordjournals.aje.a009159] [PMID] 
This Page Intentionally Left Blank 